Open Access

\title{
Enterovirus 71 infection in children with hand, foot, and mouth disease in Shanghai, China: epidemiology, clinical feature and diagnosis
}

Ying Wang ${ }^{1,3+}$, Gang Zou ${ }^{3+}$, Aimei Xia ${ }^{2+}$, Xiangshi Wang ${ }^{2}$, Jiehao Cai ${ }^{2}$, Qianqian Gao ${ }^{1,3}$, Shilin Yuan ${ }^{3}$, Guimei He ${ }^{1}$, Shuyi Zhang ${ }^{1}$, Mei Zeng ${ }^{2^{*}}$ and Ralf Altmeyer ${ }^{3^{*}}$

\begin{abstract}
Background: In 2012 a large outbreak of hand, foot, and mouth disease (HFMD) widely spread over China, causing more than 2 million cases and 567 deaths. Our purpose was to characterize the major pathogens responsible for the 2012 HFMD outbreak and analyze the genetic characterization of the enterovirus 71 (EV71) strains in Shanghai; also, to analyze the dynamic patterns of neutralizing antibody (NAb) against EV71 and evaluate the diagnostic value of several methods for clinical detection of EV71.

Methods: Clinical samples including stool, serum and CSF were collected from 396 enrolled HFMD inpatients during the peak seasons in 2012. We analyzed the molecular epidemiology, clinical feature, and diagnostic tests of EV71 infection.

Results: EV71 was responsible for $60.35 \%$ of HFMD inpatients and $88.46 \%$ of severe cases. The circulating EV71 strains belonged to subgenogroup C4a. The nucleotide sequences of VP1 between severe cases and uncomplicated cases shared 99.2 $100 \%$ of homology. Among 218 cases with EV71 infection, 211 (96.79\%) serum samples showed NAb positive against EV71 and NAb titer reached higher level 3 days after disease onset. Of 92 cases with EV71-associated meningitis or encephalitis, 5 (5.43 \%) of 92 had EV71 RNA detected in CSF samples. The blood anti-EV71 IgM assay showed a sensitivity of $93.30 \%$ and a specificity of $50 \%$.
\end{abstract}

Conclusions: EV71 C4a remained the predominant subgenotype circulating in Shanghai. The severity of the EV71 infection is not associated with the virulence determinants in VP1. RT-PCR together with IgM detection can enhance the early diagnosis of severe EV71-associated HFMD.

Keywords: Enterovirus 71, Hand, foot and mouth disease, VP1 sequence, Neutralizing antibody, EV71 diagnosis

\section{Background}

Hand, foot, and mouth disease (HFMD) has been an emerging highly contagious disease in children in countries of the Western Pacific Region since the late nineties, posing a serious public health threat. HFMD is usually a benign self-limited illness among young children

\footnotetext{
*Correspondence: zengmeigao@aliyun.com; raltmeyer@ips.ac.cn

${ }^{\dagger}$ Equal contributors

${ }^{2}$ Department of Infectious Diseases, Children's Hospital of Fudan University, Shanghai 201102, China

${ }^{3}$ Unit of Anti-infective Research, Key Laboratory of Molecular Virology \& Immunology, Institut Pasteur of Shanghai, Chinese Academy of Sciences, Shanghai 200031, China

Full list of author information is available at the end of the article
}

characterized by fever, ulcerating vesicles in the mouth and macula or papulovesicular on hands, feet and buttocks [1-4]. However, a small proportion of the affected children may develop neurological and cardiopulmonary complications with high case fatality rates $[5,6]$. The mainland China has been experiencing the persistent national outbreaks of HFMD since 2008. According to the data from the national surveillance system of HFMD in mainland China in 2008-2012, the case-fatality rate of HFMD is $0.03 \%$ and the case-severity rate is $1.1 \%$ [7]. HFMD is caused by enteroviruses belonging to the species Enterovirus A (serotypes Coxsackie virus A 2-8, 10, 12, 14,16 and enterovirus 71, 76 and 89-92), and rarely by

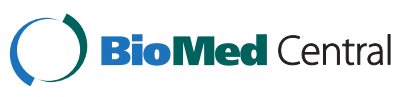


Enterovirus B, with CA16 and EV71 as the main causative agents [8, 9]. In China, EV71 infection has been associated with large outbreaks of HFMD and the majority of fatalities.

EV71, first isolated in 1969, is a single, positivelystranded RNA virus without envelope [10, 11]. The VP1 protein of EV71 contains a number of important neutralization epitopes and has been extensively used for molecular typing [12-14]. EV71 can be phylogenetically classified into 3 main genogroups (A, B and $\mathrm{C}$ ) and 11 genotypes (A, B1 $\sim \mathrm{B} 5$ and $\mathrm{C} 1 \sim \mathrm{C} 5)$ based on VP1 sequences $[15,16]$. Monitoring the genetic variations of circulating EV71 strains and the emergence of new types or recombinants of EV71 in the epidemic regions is important for vaccine development and drug discovery.

In 2012, 2,198,442 HFMD cases and 567 deathsassociated with HFMD were reported in mainland China, with the number of the notifiable cases and the fatal cases being $33.90 \%$ and $11.39 \%$ higher than those in 2011, respectively [7]. Meanwhile, the outbreaks of EV71 infection and HFMD were also reported in Malaysia, Thailand, and Cambodia in 2012 [17-19]. In this study, we collected feces, serum and CSF specimens from 396 HFMD inpatients in Shanghai during the 2012 peak season. Our purpose was to characterize the major pathogens responsible for this outbreak and to analyze the genetic characterization of the EV71 strains. Besides, we analyzed the dynamic patterns of neutralizing antibody $(\mathrm{NAb})$ titer against EV71 and evaluated the diagnostic value of several methods for the detection of EV71 in clinical samples.

\section{Results}

\section{Clinical description}

A total of 396 inpatients with HFMD including 292 (73.7 \%) uncomplicated cases and 104 (26.3\%) severe cases were enrolled into this study from March 28th to July 5th in 2012, representing $47.2 \%$ of all hospitalized HFMD cases (778) at the CHFU during the study period. The 104 severe cases included 94 (92.16\%) aseptic meningitis, 4 (3.85\%) encephalitis, 3 (2.94 \%) encephalomyelitis and 3 (2.94\%) brain stem encephalitis with neurogenic pulmonary hemorrhage/edema. Three severe cases with laboratoryconfirmed EV71 infections were fatal with 2 female infants (both were 7 months) succumbing to cardiopulmonary failure and 1 female child (5.7 years) succumbing to central respiratory failure.

Of the 396 patients, the male-to-female ratio was 1.75:1 and the age range was between 3 months and 12.7 years with the median age of 29 months old and $357(90.15 \%)$ cases $<5$ years old, of whom, 1- to 2-yearold children accounted for 127 (32.07 \%) cases. Persistent fever $>3$ days, vomiting, startle, myoclonus and higher positive rate of EV71-RNA in stool were significantly more frequent in severe cases (Table 1).

\section{EV71 detection in the stool samples by real-time RT-PCR} EV71 was identified in 239 (60.35 \%) cases, CA16 was identified in $82(20.71 \%)$ cases, EV71 and CA16 were co-detected in $3(0.758 \%)$ uncomplicated cases and the remaining $72(18.18 \%)$ cases contained untyped enteroviruses. Among the 104 severe cases, 92 (88.46\%) were positive for EV71 while only 149 (51.02 \%) positive for EV71 in 292 uncomplicated cases, revealing that EV71 was more frequently in severe cases than in uncomplicated cases $\left(\chi^{2}=45.113, \mathrm{p}<0.0001\right)$.

\section{EV71 detection in blood samples}

The average blood sampling days were $3.4 \pm 1.3$ days (range: 1-8 days) after disease onset. Among 218 cases with EV71 positivity in stool samples, 211 (96.79 \%) were positive for NAb against EV71; meanwhile, among 138 cases with EV71 negativity in stool samples, 31 (22.46 \%) were also positive for NAb against EV71. We analyzed the levels of NAb titers in sera from patients with EV71-RNA positivity in their stool samples. As shown in Fig. 1, we found that NAb GMT significantly increased with the time of disease onset $(\chi 2=46.10$, $\mathrm{p}<0.0001)$ and reached the highest level at day 6 . Although NAb GMT decreased at day 7 and day 8 after disease onset, there was no significant difference in NAb GMT at day 6, day 7 and day 8 after disease onset $(\chi 2=1.912, \mathrm{p}=0.385)$.

Of 200 serum samples obtained from the patients with EV71 RNA positive in stool samples and positive NAb against EV71, 151 (75.5 \%) were positive for EV71 RNA in sera. Serum EV71 RNA was detected positive in 44 $(60.27 \%)$ of 73 severe cases and $107(84.25 \%)$ of 127 uncomplicated cases, respectively $(\chi 2=14.41, \mathrm{p}=0.0001)$. The NAb GMT was 1122.5 and 1622.6 in 73 severe cases and 127 uncomplicated cases, respectively $(\chi 2=4.084$, $\mathrm{p}=0.043$ ), indicating there was no difference in the NAb titer level between severe cases and uncomplicated cases. The average serum sampling days after disease onset was $3.18 \pm 1.04$ days (range: $1-5$ days) in severe cases and $3.9 \pm 1.34$ days in uncomplicated cases (range: $1-8$ days) $(x 2=15.90, \mathrm{p}<0.0001)$, respectively.

Of the enrolled 396 patients, 267 who had both EV71-RNA detected in stool samples and NAb detected in serum samples had EV71 IgM tested in whole blood samples from finger tip. Compared to the EV71 infection confirmed by both positive EV71-RNA in stool and positive serum NAb against EV71, blood EV71 IgM showed a sensitivity of $93.30 \%$ and a specificity of $50 \%$; the positive predict value was $79.15 \%$ and the negative predict value was $78.57 \%$ (Table 2).

\section{Phylogenetic analyses of VP1 gene of EV71}

The entire VP1 region of EV71 was amplified using RTPCR directly from the clinical serum and CSF specimens 
Table 1 Comparison of clinical manifestations between uncomplicated cases and severe cases

\begin{tabular}{|c|c|c|c|c|c|}
\hline & $\begin{array}{l}\text { All cases } \\
(396)\end{array}$ & $\begin{array}{l}\text { Uncomplicated } \\
(73.74 \%, 292)\end{array}$ & $\begin{array}{l}\text { Severe } \\
(26.26 \%, 104)\end{array}$ & $x^{2}$ & $P$ value $^{*}$ \\
\hline Male to female ratio & $1.75(252 / 144)$ & $1.92(192 / 100)$ & $1.36(60 / 44)$ & 2.153 & 0.155 \\
\hline Fever $>3$ days & $16.41 \%(65)$ & $11.64 \%(34)$ & $29.81 \%(31)$ & 18.441 & $<0.0001$ \\
\hline Oral rash & $88.38 \%$ (350) & $89.38 \%(261)$ & $85.58 \%(89)$ & 1.082 & 0.291 \\
\hline Vomiting & $45.45 \%(180)$ & $38.70 \%(113)$ & $64.42 \%(67)$ & 20.468 & $<0.0001$ \\
\hline Headache & $2.53 \%(10)$ & $2.05 \%(6)$ & $3.85 \%(4)$ & 1.000 & 0.298 \\
\hline Startle & $57.32 \%(227)$ & $46.92 \%(137)$ & $86.54 \%(90)$ & 49.209 & $<0.0001$ \\
\hline Convulsion & $1.26 \%(5)$ & $0.68 \%(2)$ & $2.88 \%(3)$ & 2.976 & 0.116 \\
\hline Myoclonus & $35.10 \%(139)$ & $21.92 \%(64)$ & $72.12 \%(75)$ & 84.826 & $<0.0001$ \\
\hline EV71-RNA positive in stool & 242 (61.11\%) & $143(48.97 \%)$ & 99 (95.19\%) & 68.933 & $<0.0001$ \\
\hline
\end{tabular}

${ }^{*}$ Chi-squared test was used to evaluate the significant variation of clinical manifestations between uncomplicated cases and severe cases. The $\mathrm{p}$ value less than 0.05 was considered to be significant and marked in bold

rather than from cultured isolates to avoid adaptive mutations selected during serial passage. EV71 RNA was detected in $5(5.43 \%)$ of 92 CSF samples obtained from the severe patients with EV71 RNA positive in the stool samples. The PCR products of 151 EV71-positive serum samples and 5 EV71-positive CSF samples could be sequenced successfully. The EV71 VP1 nucleotide and amino acid sequence among the 2012 Shanghai strains showed $99.2 \sim 99.8 \%$ and $98.9 \sim 99.6 \%$ identity, respectively. Based on the VP1 sequences, the nucleotide and amino acid homologies between severe cases and uncomplicated cases were $99.2 \sim 100 \%$ and $98.9 \sim 100 \%$, respectively.

A phylogenetic tree was constructed based on VP1 sequences to determine the subgroups and subgenotypes of EV71 strains. Seventy reference sequences were obtained from GenBank, including 11 subgenotypes (A, B1-B5, and C1-C5) and representative strains epidemic in China since 2007. In addition, another twenty-six VP1 sequences of EV71 from 2008 to 2011 isolated in Shanghai

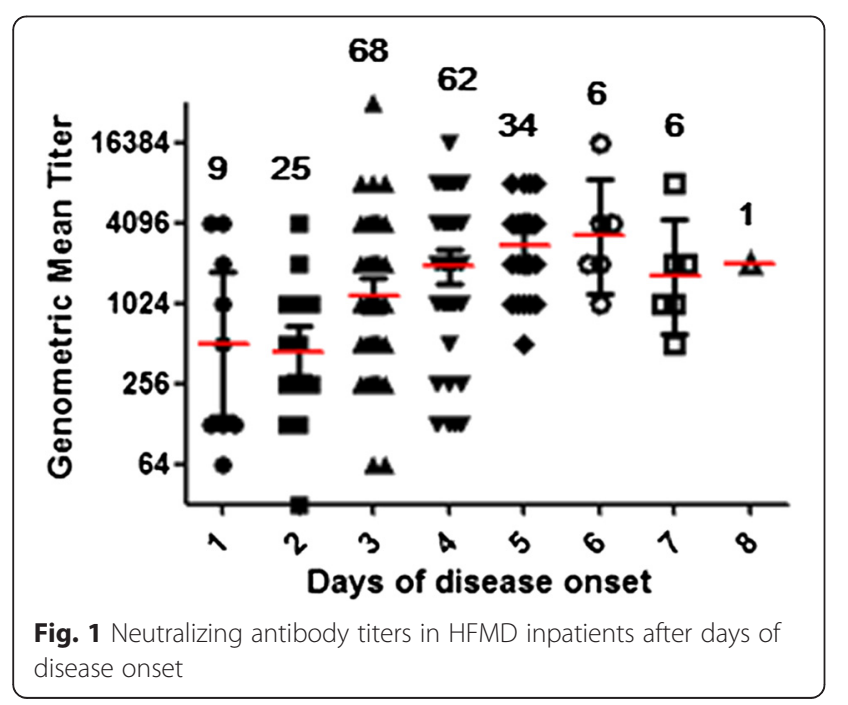

were also used for alignment analyses. As shown in Fig. 2, all the Shanghai sequences were classified into the C4a cluster which was predominant since the 2008 HFMD outbreak in mainland China. The 2012 Shanghai EV71 strains shared a high degree of nucleotide homology (98.5 99.8 \%) with 2008 Anhui strain 18.08/AH/ $\mathrm{CHN} / 2008$. It should be noted that the nine 2012 Shanghai strains formed a separated subcluster (99\% bootstrap value) of the C4a clade from the 2011 Shanghai strains fell within the same cluster as the EV71 strains isolated from Guangdong, Shandong and Anhui regions in China during 2008-2009, sharing $96.63 \sim 98.88 \%$ nucleotide and $98.32 \sim 99.66 \%$ amino acid homologies, respectively. The VP1 sequences of 2012 EV71 strains circulating in Shanghai shared 93.94-99.33\% nucleotide and 96.97-98.99 \% amino acid homologies with the 2008-2011 strains.

\section{Discussion}

Although multiple genotypes and subgenotype of EV71 are circulating simultaneously or alternatively in other countries and regions, [20,21] our study revealed that EV71 remained the predominant enterovirus causing the 2012 large outbreaks of HFMD and severe central nerves system (CNS) disease in Shanghai. Combined with our previous surveillance data, $[22,23]$ we reason that the principal strategy of controlling the HFMD outbreak in Shanghai is to prevent the EV71 circulation in children through mass EV71 vaccination. In line with previous

Table 2 Evaluation of blood EV71 IgM rapid test kit

\begin{tabular}{lccc}
\hline $\begin{array}{l}\text { Blood EV71-IgM } \\
\text { antibody }\end{array}$ & \multicolumn{3}{c}{ stool EV71-RNA and serum NAb against EV71 } \\
& Both positive & Both negative & Total \\
\hline Positive & 167 & 44 & 211 \\
Negative & 12 & 44 & 56 \\
Total & 179 & 88 & 267 \\
\hline
\end{tabular}




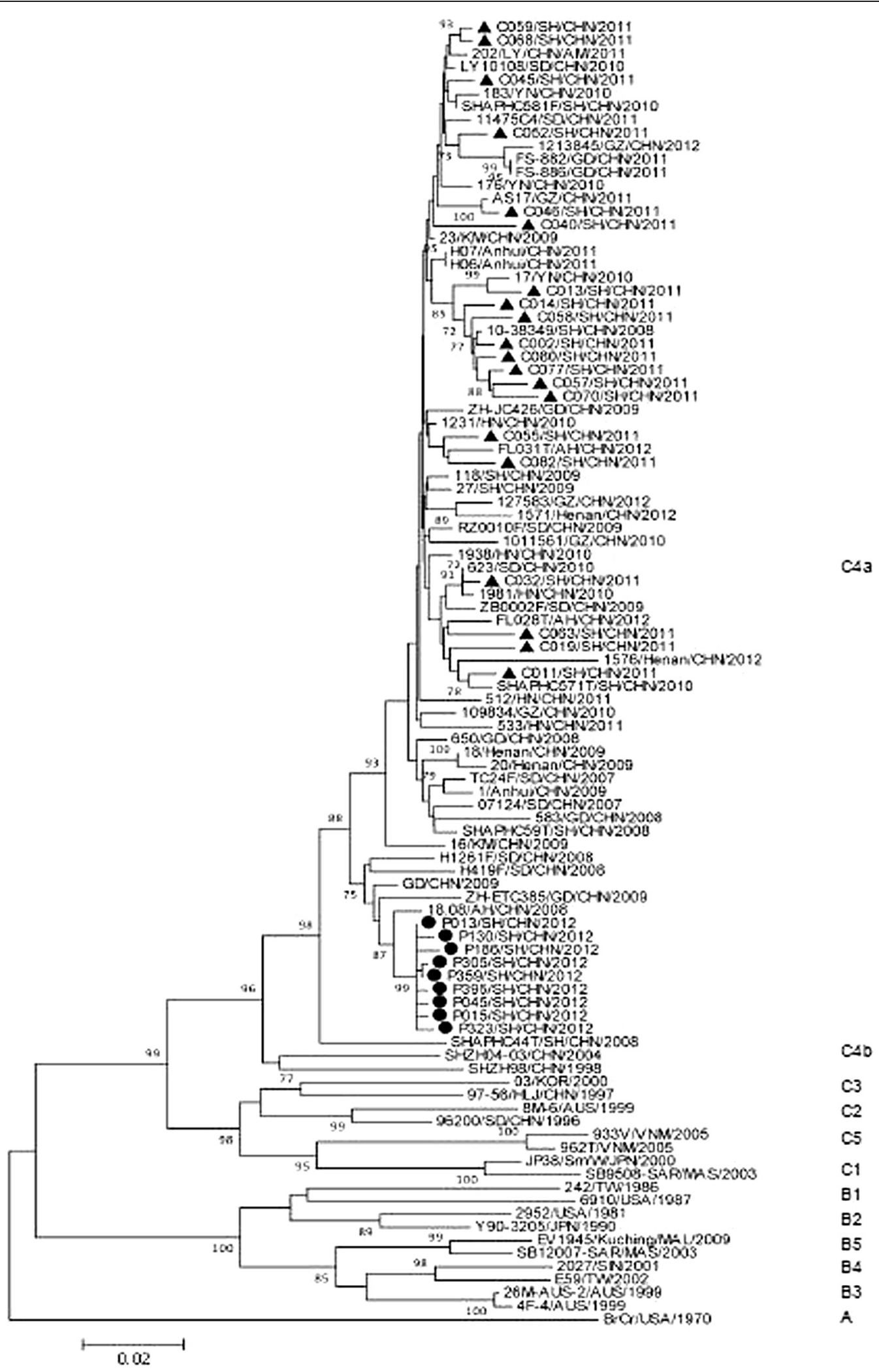

Fig. 2 (See legend on next page.) 
(See figure on previous page.)

Fig. 2 Phylogenetic analysis of EV71 from Shanghai, 2012. The phylogenetic tree was based on the alignment of entire VP1 sequences using Neighbor-Joining method with bootstrap value 1000 pseudoreplicates. Bootstrap values with $>75$ replications were shown at the branch nodes. The scale bar represented the genetic distance of 0.02 nucleotide substitutions per site. $\boldsymbol{\Delta}$ indicated EV71 strains from Shanghai, 2011 , $\bullet$ indicated EV71 strains from Shanghai, 2012. Other strains are obtained from GenBank (listed in Additional file 1)

studies, we also found that persistent fever $>3$ days, vomiting, startle, and myoclonus can be used as the warning signs for predicting the severe HFMD cases [8, 24].

We noted that the VP1 sequences of EV71 strains from severe cases were indistinguishable from those causing uncomplicated symptoms, which is same as other reports $[11,25,26]$. Besides, the VP1 sequences of EV71 strains in the serum and CSF specimens collected from the same patients were identical, suggesting the individual patient appeared to be infected with a single genotype of EV71. Phylogenetic analysis suggests that the constant genetic evolution of the EV71 strains circulating in Shanghai occurred in the VP1 gene over the year. It deserves to further explore the effect of the genetic evolution of VP1 on the antigen change of EV71 [27, 28]. Previous studies have reported that the residue L97 and domain 215-KQEKD-219 on VP1 protein of EV71 were associated with neutralizing epitope. We found that these neutralizing epitopes were unchanged in all the Shanghai strains, making the development and long-term use of EV71 vaccine feasible $[29,30]$.

Neutralization antibody plays an important role in immunoprotection from viral infection as it can efficiently bind virus, neutralize virus, and thus prevent the further progression of disease. It is usually believed that NAb produces and reaches the peak level during the late convalescent stage of viral infection. Based on these theories, IVIG containing NAb against EV71 is recommended for treating the severe EV71-associated diseases in many countries including our countries $[21,31]$. Clinical observational study from Malaysia showed early IVIG therapy in severe EV71-associated HFMD patients might have a beneficial effect on halting disease progression and improve the outcomes [32]. Also, a mice model study showed that plasma containing high titer EV71-sepcific IVIG products can confer protection against lethal EV71 challenge [33]. However, we observed that serum NAb against EV71 has produced in over $95 \%$ of EV71-infected HFMD patients when the HFMD-related symptoms appear and reaches the high level within 3-6 days after disease onset. In clinical setting, severe cases were admitted to hospital usually on day 3 after disease onset when NAb has already reached the high level. Therefore, our data suggest that if IVIG has any benefit in the treatment of HFMD, it is likely not due to the direct antivrial effect of EV71 neutralizing Abs. An immunomodulatory effect of IVIG has been proposed by In vitro data [34].
We detected 5 EV71 strains in 92 CSF samples using RT-PCR method and obtained the sequences of EV71. However, EV71 could not be isolated from CSF samples after 5 passages in RD cells. Obviously, CSF samples are not suitable to be used as the prior clinical samples for the diagnosis of EV71-associated CNS infections. Other studies reported the difficulty in isolating EV71 from CSF due to low amount of viral load, sensitivity of PCR, and intrathecal antibody in CSF [5, 22, 35, 36]. Real-time RT-PCR and viral isolation are considered as the standard methods in clinical diagnosis of viral infection. Although real-time RT-PCR has the advantage of high sensitivity and specificity in the diagnosis of EV71 infection in clinical samples, it takes $2-3 \mathrm{~h}$ to get the result and requires the expensive equipment in the hospital laboratory. Also, virus isolation is time-consuming and insensitive. The fatal HFMD was almost always caused by EV71 infection and the severe disease progress is very fast, thus, timely and rapid diagnosis of EV71 infection for suspected severe HFMD is critical for clinical management and early intervention [37]. Thus, a rapid, sensitive and convenient bedside diagnostic tool is needed in clinical practice. Blood EV71-IgM-Colloidal Gold immunochromatographic assay is easy to perform and provides the result within $15 \mathrm{~min}$. It has high sensitivity and negative predict value but low specificity and positive predict value, therefore, EV71-IgM-Colloidal Gold assay as the confirmatory test for diagnosing EV71 infection was not recommended. However, given the high sensitivity and convenience, finger tip blood EV71 IgM test can be used as a rapid point-of-care screen tool and an essential supplement for the early diagnosis of EV71 infection to help clinicians timely recognize severe EV71-associated diseases.

The upcoming EV71 vaccination in China is a promising strategy to prevent the large outbreak of HFMD. Early recognition of progressive symptoms and timely diagnosis of EV71 infection in suspected severe HFMD will help take appropriate clinical intervention to reduce the fatality through intensive care. Ongoing surveillance of HFMD and EV71 infections and evolution of EV71 strains is necessary for control of HFMD in China and the formulation of EV71 vaccine before and after vaccine available in the market.

\section{Conclusions}

This study revealed that EV71 remained the predominant enterovirus causing the 2012 large outbreaks of HFMD and severe CNS disease in Shanghai. We observed the 
constant genetic evolution of EV7 VP1 over the year. In fact, serum NAb against EV71 in HFMD patients has reached higher level 3 days after disease onset. Real-time RT-PCR together with IgM detection would enhance the detection of HFMD and thereby should be highly recommended in clinical therapy. Our findings provide important epidemiological information of HFMD epidemic in Shanghai and evaluate the issues regarding the laboratory diagnosis of EV71 infection.

\section{Methods}

\section{Specimen and data collection}

Clinical specimens used in this study were collected from hospitalized HFMD patients during the 2012 peak season (March 28th - July 5th) of HFMD in Shanghai at the Children's Hospital of Fudan University (CHFU). The study was approved by the Ethics Committee of the study hospital. Both stool samples and serum samples were collected within $24 \mathrm{~h}$ after admission and a CSF sample was collected if a patient was highly suspected to have central nervous involvement and had a successful lumbar puncture procedure for the routine CSF diagnostic tests. A severe case was defined by HFMD accompanied with at least one of the following complications: aseptic meningitis, encephalitis, acute flaccid paralysis, pulmonary edema or hemorrhage, cardiopulmonary collapse. CSF pleocytosis was defined as a white blood cell count more than $10 \times 10^{6}$ cells/L in a patient older than 1 month of age. A total of 263 serum specimens were collected from 292 uncomplicated HFMD patients, and 93 serum and 92 CSF specimens were collected from 104 severe HFMD patients. All the samples were stored at $-80^{\circ} \mathrm{C}$ for further tests. Clinical data were recorded using casereport form (CRF) and re-assessed by an infectious disease specialist.

\section{Detection of EV71 infection in clinical laboratory}

EV71 detection in stool specimens was performed using commercially available pan-enterovirus, EV71, and CA16 diagnostic kit (Da An Gene Co., Ltd, Guangzhou, China) according to manufacturer's instructions. Anti-EV71 IgM was detected in the peripheral finger tip blood sample using EV71-IgM-Colloidal Gold immunochromatographic assay (Beijing Wantai Biological Pharmacy Enterprise Co., Ltd., China).

RT-PCR detection, virus isolation, and neutralization assay Viral RNA was extracted from serum and CSF specimens using a QIAamp Viral RNA Mini Kit (Qiagen, Hilden, Germany). Direct RT-PCR was performed using SuperScript III One-Step System with Platinum Taq High Fidelity (Invitrogen, USA) with primer targeting EV71 VP1 gene (2372 F: 5'-GCAGCCCAAAA GAACTTCAC-3', and 3454R: 5'-AAGTCGCGAGAGC
TGTCTTC-3') [1]. The resulting 1083-bp PCR product was analyzed on a $1 \%$ agarose gel, and then purified using TIANgel Midi Purification Kit (TIANGEN, China) for sequencing. Of 92 CSF samples, 5 were positive using direct RT-PCR. However, virus isolation for 92 CSF samples using the strategy described in previous study was unsuccessful [38]. Neutralization assay was performed using the method described in previous study [39].

\section{Sequencing of EV71 strains and phylogenetic analyses}

The gel purified PCR products were bi-directionally sequenced using an ABI 3730xl automatic DNA analyzer. Alignment of the entire VP1 nucleotide sequences (891 bp) of the EV71 strain was performed using Clustal W program. Phylogenetic tree was constructed in MEGA 5.2 (Molecular Evolutionary Genetics Analysis software; Tamura, Dudley, Nei, and Kumar 2012) using Neighbor-joining method in Kimura two-parameter model, accompanied by bootstrap analyses with 1000 replicates. The entire VP1 nucleotide sequences of the nine Shanghai EV71 strains and reference sequences obtained from GenBank were listed with accession numbers in Additional file 1.

\section{Statistical analyses}

The data were analyzed using Microsoft Excel 2010, SPSS software version 19.0 and GraphPad Prism V5.01 software. The percentage of clinical data was analyzed using the chi-square test. NAb titers were log-transformed to geometric mean titers (GMT) for statistical analysis. GMT and the mean sampling days were analyzed using Kruskal Wallis test. The p value less than 0.05 was considered to be statistically significant.

\section{Additional file}

Additional file 1: The list of EV71 strains used for phylogenetic analysis in this study.

Competing interests

All authors declare that they have no competing interests.

Authors' contributions

AX, XW, JC carried out the specimen collection, clinical data record and real-time RT-PCR. YW, QG, SY carried out the molecular genetic studies, participated in the sequence alignment. YW participated in the neutralization and performed the statistical analysis. YW, MZ and GZ drafted the manuscript. MZ, RA, GZ, GH and SZ participated in the design of the study. All authors read and approved the final manuscript.

\section{Acknowledgement}

This study was supported by Pasteur International Network Association. We thank Dr. Chiyu Zhang from Pathogen Diagnostic Center, Institut Pasteur of Shanghai for thoughtful comments and review of the manuscript. 


\section{Author details}

${ }^{1}$ Institute of Molecular Ecology and Evolution, SKLEC \& IECR, East China Normal University, Shanghai 200062, China. ${ }^{2}$ Department of Infectious Diseases, Children's Hospital of Fudan University, Shanghai 201102, China. ${ }^{3}$ Unit of Anti-infective Research, Key Laboratory of Molecular Virology \& Immunology, Institut Pasteur of Shanghai, Chinese Academy of Sciences, Shanghai 200031, China.

Received: 2 March 2015 Accepted: 8 May 2015

Published online: 03 June 2015

\section{References}

1. Zhang Y, Tan XJ, Wang HY, Yan DM, Zhu SL, Wang DY, et al. An outbreak of hand, foot, and mouth disease associated with subgenotype C4 of human enterovirus 71 in Shandong, China. J Clin Virol. 2009;44:262-7.

2. Chong CY, Chan KP, Shah VA, Ng WY, Lau G, Teo TE, et al. Hand, foot and mouth disease in Singapore: a comparison of fatal and non-fatal cases. Acta Paediatr. 2003:92:1163-9.

3. Ho M, Chen ER, Hsu KH, Twu SJ, Chen KT, Tsai SF, et al. An epidemic of enterovirus 71 infection in Taiwan. Taiwan Enterovirus Epidemic Working Group. N Engl J Med. 1999;341:929-35.

4. Sabanathan S, Tanle V, Thwaites L, Wills B, Qui PT, RogiervanDoorn H. Enterovirus 71 related severe hand, foot and mouth disease outbreaks in South-East Asia: current situation and ongoing challenges. J Epidemiol Community Health. 2014;68:500-2.

5. Chang LY, Lin TY, Hsu KH, Huang YC, Lin KL, Hsueh C, et al. Clinical features and risk factors of pulmonary oedema after enterovirus-71-related hand, foot, and mouth disease. Lancet. 1999;354:1682-6.

6. Chan LG, Parashar UD, Lye MS, Ong FG, Zaki SR, Alexander JP, et al. Deaths of children during an outbreak of hand, foot, and mouth disease in sarawak, malaysia: clinical and pathological characteristics of the disease. For the Outbreak Study Group. Clin Infect Dis. 2000;31:678-83.

7. Xing W, Liao Q, Viboud C, Zhang J, Sun J, Wu JT, et al. Hand, foot, and mouth disease in China, 2008-12: an epidemiological study. Lancet Infect Dis. 2014;14:308-18.

8. Liu W, Wu S, Xiong Y, Li T, Wen Z, Yan M, et al. Co-Circulation and Genomic Recombination of Coxsackievirus A16 and Enterovirus 71 during a Large Outbreak of Hand, Foot, and Mouth Disease in Central China. PLoS One. 2014;9, e96051.

9. Yip CC, Lau SK, Zhou B, Zhang MX, Tsoi HW, Chan KH, et al. Emergence of enterovirus 71 "double-recombinant" strains belonging to a novel genotype D originating from southern China: first evidence for combination of intratypic and intertypic recombination events in EV71. Arch Virol. 2010;155:1413-24

10. Schmidt RC, Maassab HF. Local immunity to influenza virus in chicken tracheal organ cultures. J Infect Dis. 1974;129:637-43.

11. Zhang Y, Zhu Z, Yang W, Ren J, Tan X, Wang Y, et al. An emerging recombinant human enterovirus 71 responsible for the 2008 outbreak of hand foot and mouth disease in Fuyang city of China. Virol J. 2010;7:94.

12. Oberste MS, Maher K, Kilpatrick DR, Flemister MR, Brown BA, Pallansch MA. Typing of human enteroviruses by partial sequencing of VP1. J Clin Microbiol. 1999;37:1288-93.

13. Oberste MS, Maher K, Kilpatrick DR, Pallansch MA. Molecular evolution of the human enteroviruses: correlation of serotype with VP1 sequence and application to picornavirus classification. J Virol. 1999;73:1941-8.

14. Caro V, Guillot S, Delpeyroux F, Crainic R. Molecular strategy for 'serotyping of human enteroviruses. J Gen Virol. 2001:82:79-91.

15. Brown BA, Oberste MS, Alexander Jr JP, Kennett ML, Pallansch MA. Molecular epidemiology and evolution of enterovirus 71 strains isolated from 1970 to 1998. J Virol. 1999;73:9969-75.

16. Akhmadishina LV, Eremeeva TP, Trotsenko OE, Ivanova OE, Mikhailov MI, Lukashev AN. Seroepidemiology and molecular epidemiology of enterovirus 71 in Russia. PLoS One. 2014;9, e97404.

17. Sham NM, Krishnarajah I, Ibrahim NA, Lye MS. Temporal and spatial mapping of hand, foot and mouth disease in Sarawak, Malaysia. Geospat Health. 2014;8:503-7.

18. Puenpa J, Mauleekoonphairoj J, Linsuwanon P, Suwannakarn K, Chieochansin T, Korkong S, et al. Prevalence and Characterization of Enterovirus Infections among Pediatric Patients with Hand Foot Mouth Disease, Herpangina and Influenza Like IIIness in Thailand, 2012. PLoS One. 2014;9, e98888.
19. Seiff A. Cambodia unravels cause of mystery illness. Lancet. 2012;380:206

20. Solomon T, Lewthwaite P, Perera D, Cardosa MJ, McMinn P, Ooi MH. Virology, epidemiology, pathogenesis, and control of enterovirus 71. Lancet Infect Dis. 2010;10:778-90

21. Organization WH. A Guide to Clinical Management and Public Health Response for Hand, Foot and Mouth Disease (HFMD). 2009.

22. Zeng M, Li YF, Wang XH, Lu GP, Shen HG, Yu H, et al. Epidemiology of hand, foot, and mouth disease in children in Shanghai 2007-2010. Epidemiol Infect. 2012;140:1122-30.

23. Xu Z, Shen H, Wang Z, Altmeyer R, Xia A, Mao S, et al. The population-based health effect of hand, foot and mouth disease in children in Shanghai. Pediatr Infect Dis J. 2014;33:448-52.

24. Zhang Q, Macdonald NE, Smith JC, Cai K, Yu H, Li H, et al. Severe enterovirus type 71 nervous system infections in children in the shanghai region of china: clinical manifestations and implications for prevention and care. Pediatr Infect Dis J. 2014;33:482-7.

25. Singh S, Poh CL, Chow VT. Complete sequence analyses of enterovirus 71 strains from fatal and non-fatal cases of the hand, foot and mouth disease outbreak in Singapore (2000). Microbiol Immunol. 2002:46:801-8.

26. Shih SR, Ho MS, Lin KH, Wu SL, Chen YT, Wu CN, et al. Genetic analysis of enterovirus 71 isolated from fatal and non-fatal cases of hand, foot and mouth disease during an epidemic in Taiwan, 1998. Virus Res. 2000;68:127-36.

27. Kung SH, Wang SF, Huang CW, Hsu CC, Liu HF, Yang JY. Genetic and antigenic analyses of enterovirus 71 isolates in Taiwan during 1998-2005. Clin Microbiol Infect. 2007;13:782-7.

28. Huang SW, Hsu YW, Smith DJ, Kiang D, Tsai HP, Lin KH, et al. Reemergence of enterovirus 71 in 2008 in taiwan: dynamics of genetic and antigenic evolution from 1998 to 2008. J Clin Microbiol. 2009;47:3653-62.

29. Cordey S, Petty TJ, Schibler M, Martinez Y, Gerlach D, van Belle S, et al. Identification of site-specific adaptations conferring increased neural cell tropism during human enterovirus 71 infection. PLoS Pathog. 2012;8, e1002826.

30. Kiener TK, Jia Q, Meng T, Chow VT, Kwang J. A novel universal neutralizing monoclonal antibody against enterovirus 71 that targets the highly conserved "knob" region of VP3 protein. PLoS Negl Trop Dis. 2014;8, e2895.

31. Wu CY, Wang HC, Wang KT, Weng SC, Chang WH, Shih DY, et al. Neutralization of five subgenotypes of Enterovirus 71 by Taiwanese human plasma and Taiwanese plasma derived intravenous immunoglobulin. Biologicals. 2013;41:154-7.

32. Ooi MH, Wong SC, Podin Y, Akin W, del Sel S, Mohan A, et al. Human enterovirus 71 disease in Sarawak, Malaysia: a prospective clinical, virological, and molecular epidemiological study. Clin Infect Dis. 2007:44:646-56

33. Cao RY, Han JF, Jiang T, Tian X, Yu M, Deng YQ, et al. In vitro and in vivo characterization of a new enterovirus type 71-specific human intravenous immunoglobulin manufactured from selected plasma donors. J Clin Virol. 2011:51:246-9.

34. Wang SM, Lei HY, Huang MC, Su LY, Lin HC, Yu CK, et al. Modulation of cytokine production by intravenous immunoglobulin in patients with enterovirus 71-associated brainstem encephalitis. J Clin Virol. 2006;37:47-52.

35. Perez-Velez CM, Anderson MS, Robinson CC, McFarland EJ, Nix WA, Pallansch MA, et al. Outbreak of neurologic enterovirus type 71 disease: a diagnostic challenge. Clin Infect Dis. 2007;45:950-7.

36. Wang JR, Tsai HP, Chen PF, Lai YJ, Yan JJ, Kiang D, et al. An outbreak of enterovirus 71 infection in Taiwan, 1998. II. Laboratory diagnosis and genetic analysis. J Clin Virol. 2000;17:91-9.

37. Ooi MH, Wong SC, Lewthwaite P, Cardosa MJ, Solomon T. Clinical features, diagnosis, and management of enterovirus 71. Lancet Neurol. 2010;9:1097-105

38. Wang $Y$, Zhu Q, Zeng M, Altmeyer R, Zou G. Complete genome sequence of a human enterovirus 71 strain isolated from a fatal case in Shanghai, China, in 2012. Genome Announc. 2014;2:e00457-14.

39. Zeng M, El Khatib NF, Tu S, Ren P, Xu S, Zhu Q, et al. Seroepidemiology of Enterovirus 71 infection prior to the 2011 season in children in Shanghai. J Clin Virol. 2012;53:285-9. 